\title{
Problématique du contrôle et de la prévention de la coccidiose du poulet
}

\author{
Hervé Brice DAKPOGAN ${ }^{1 *}$, Sahidou SALIFOU ${ }^{1}$, Guy Apolinaire MENSAH ${ }^{2}$, \\ Armand GBANGBOTCHE ${ }^{3}$, Issaka YOUSSAO ${ }^{1}$, Muriel NACIRI ${ }^{4}$ et Nestor SAKITI $^{5}$

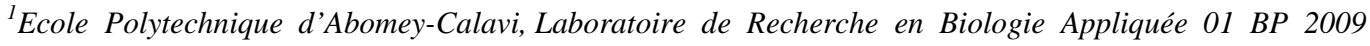 \\ Cotonou, Bénin. \\ ${ }^{2}$ Centre de Recherche Agricole d'Agonkanmey, Institut National de Recherche Agricole du Bénin, 01 \\ BP 988 Abomey-Calavi, Bénin. \\ ${ }^{3}$ Département de Production Animale de la Faculté des Sciences Agronomiques, 01 BP 526 \\ Cotonou, Bénin. \\ ${ }^{4}$ INRA - UR 86 Bio-Agresseurs Santé et Environnement, 37380 Nouzilly, France. \\ ${ }^{5}$ Laboratoire de Parasitologie et d'Ecologie Parasitaire, Faculté des Sciences et Techniques 01 BP \\ 4521 Cotonou, Bénin. \\ *Auteur correspondant, E-mail: dakpogan2002@yahoo.fr; Tel: (00229)94 625836.
}

\section{RESUME}

La coccidiose chez le poulet est une pathologie digestive causée par les sept espèces du genre Eimeria dont les plus pathogènes sont: E. tenella, E. acervulina, E. brunetti et E. maxima. Le cycle de vie des coccidies est direct et très court souvent réalisé en sept jours et qui débouche sur la formation des oocystes excrétés à travers les fèces. L'incidence économique de la maladie est estimée à 2,3 milliards d'Euro mondialement avec $70 \%$ des pertes attribuables à la coccidiose sub-clinique, difficilement perceptible, qui déprime le gain de poids vif corporel et l'indice de consommation alimentaire du poulet. L'utilisation du PCR (Polymerase Chain Reaction) permet d'identifier les espèces de coccidies par l'analyse du génome. Les mesures de prévention et de contrôle sont basées sur l'utilisation des anticoccidiens et des vaccins. Toutefois, les problèmes de résistance des coccidies aux médicaments, la présence de résidus médicamenteux dans les produits avicoles et la forme sub-clinique de la maladie engendrée par la réplication des coccidies vaccinales dans les entérocytes, constituent de graves menaces pour la filière poulet. D'autres moyens de lutte continuent de faire l'objet d'expérimentation à travers les plantes médicinales, et les vaccins recombinés. L'utilisation de la résistance naturelle aux coccidies de certains génotypes de poulet est une perspective envisageable qui peut définitivement mettre l'aviculture à l'abri des pertes énormes engendrées par les mesures de contrôle actuelles et la forme sub-clinique de la maladie.

(c) 2012 International Formulae Group. All rights reserved.

Mots clés: Eimeria, anticoccidien, vaccin, plante médicinale, résistance naturelle.

\section{INTRODUCTION}

La coccidiose est une maladie parasitaire rencontrée chez les animaux, comme les volailles, mammifères, rongeurs et lagomorphes, etc. et causée par un protozoaire du phylum des Apicomplexa, de la famille des Eimeridae et du genre Eimeria. Sept espèces d'importance 
pathologique sont rencontrées chez le poulet: Eimeria acervulina, Eimeria brunetti, Eimeria maxima, Eimeria necatrix, Eimeria tenella, Eimeria praecox et Eimeria mitis (Ovington et al., 1995). Les coccidies sont présentes dans le milieu extérieur sous forme de spore entourée d'une coque assez résistante appelée oocyste (Williams, 1999). Les oocystes deviennent infectieux deux jours après l'excrétion et sont ingérés directement par les poulets sains. Le cycle évolutif des coccidies de poulet est direct sans l'intervention d'un hôte intermédiaire. La coccidiose est une maladie qui se manifeste par les signes digestifs dont le plus important est la diarrhée qui peut devenir sanguinolente au niveau de certaines espèces de coccidies. La multiplication des sporozoïtes dans le tractus intestinal entraîne la lésion du tissu épithélial, avec l'interruption de la nutrition, du processus digestif et de l'absorption des nutriments (McDougald, 2003). Il en résulte de la déshydratation et de la perte de sang. Les dégâts organiques causés par les coccidies, constituent des conditions favorables d'expression et de développement de certaines bactéries gastro-intestinales telles que les clostridies, les salmonelles et les colibacilles (Bostvironnois et Zadjian, 2011). La coccidiose est également exacerbée par certaines maladies virales immunosuppressives telles que : la boursite infectieuse, la maladie de Marek et l'anémie virale du poulet (Lanckriert et al., 2010).

Le cycle direct des coccidies les rend omniprésentes dans tous les systèmes d'élevages avicoles. Le développement de l'aviculture moderne avec la claustration totale des oiseaux est un succès en partie attribuable au contrôle de la coccidiose. Ce contrôle est l'opération la plus coûteuse en aviculture moderne (Williams, 1999) et est caractérisé par l'utilisation de certains principes actifs comme des additifs alimentaires à faible dose à titre préventif et à dose plus élevée à titre curatif dans l'aliment ou l'eau de boisson. Sørensen et al. (2006) ont estimé par une extrapolation le coût total des pertes engendrées par la coccidiose clinique, sa forme sub-clinique et son contrôle à plus de 2,3 milliards d'Euro mondialement, avec $70 \%$ des pertes attribuables à la coccidiose sub-clinique inapparente qui déprime considérablement le gain de poids vif corporel et affecte l'indice de consommation. La résistance des coccidies aux anticoccidiens constitue déjà une préoccupation majeure des industries avicoles. De même, la présence de résidus médicamenteux dans les produits et sous produits de la volaille est préjudiciable à la santé des consommateurs (Cannavan et al., 2000; Mortier et al., 2005; Danaher et al., 2008). Le but de cette synthèse bibliographique, est de faire le point des différents modes développés pour la prévention et le contrôle de la coccidiose du poulet, d'évaluer les limites de ces différents moyens de contrôle et de prévention et enfin, de mettre l'accent sur les méthodes alternatives envisageables en occurrence, l'utilisation de la résistance naturelle de certaines souches et génotypes de poulet, pour une réduction considérable et durable des pertes causées par cette maladie.

\section{Étiologie de la coccidiose du poulet}

Les protozoaires sont historiquement placés dans un seul embranchement contenant tous les animaux unicellulaires. L'organisation complexe et la diversification structurelle des protozoaires ont conduit à la séparation des classes en sept ordres différents (Levine, 1985). Deux de ces ordres comportent des espèces qui sont des parasites importants de la volaille. L'ordre des Sarcomastigophora qui sont les flagellés et les amibes, possédant généralement des pseudopodes ou flagelles 
ou les deux, comme des organelles locomoteurs. Les genres reconnus importants pour la volaille dans cet ordre sont: Histomonas, Trypanosoma, Chilomastix, Entamoeba, Endolimax et Hexamita (Reetz, 1993). Le second est l'ordre des Apicomplexa strictement intracellulaire et caractérisé par la présence d'un complexe apical dans le sporozoïte avec les genres comme Eimeria, Isospora, Haemoproteus, Leucocytozoon, Plasmodium, Toxoplasma, Sarcocystis, Wenyonella, Tyzzeria et Cryptosporidium (McDougald, 2003). La coccidiose est causée par le genre Eimeria. Des sept espèces décrites que sont Eimeria tenella, E. necatrix, E. brunetti, E. maxima, E. acervulina, E. mitis et $E$. praecox (Ovington et al., 1995), cinq sont véritablement pathogènes $(E$. tenella, $E$. acervulina, E. necatrix, E. maxima et $E$. brunetti) et entraînent un état morbide assez sévère chez l'oiseau. L'oocyste de Eimeria est un zygote à coque externe épaisse, excrété dans les fèces de l'hôte infecté et qui sporule 48 heures après l'excrétion (Long, 1982). De nos jours, l'analyse morphométrique sous microscope optique et mieux l'utilisation du PCR (Polymerase Chain Réaction), permettent une identification rapide et qualitative des espèces de Eimeria (Vancraeynest et al., 2011).

\section{Biologie et cycle de développement des coccidies}

Le cycle de vie des coccidies est un cycle direct et très court, souvent réalisé en sept jours comme le montre la Figure 1 (McDougald, 2003), comparativement aux helminthes qui ont un cycle long. Les oocystes sont excrétés dans les fèces et sporulent entre 1 et 2 jours. L'oocyste est l'élément de dissémination et l'élément de résistance dans le milieu extérieur, c'est aussi la fin et le début du cycle évolutif.

\section{Développement endogène}

Les coccidies diffèrent des bactéries et des virus dans le caractère auto limitatif de développement de la maladie qu'ils provoquent. Après l'absorption orale des oocystes, ceux-ci sont écrasés dans le gésier. Les sporozoïtes éclosent dans le petit intestin à partir des sporocystes (2-6) . Ils entrent dans la muqueuse intestinale et entament le cycle conduisant à la reproduction. Après la pénétration des muqueuses, les schizontes de première génération multinucléaires sont formés (3) dans la vacuole parasitophore (PV). Les schizontes produisent des mérozoïtes de première génération motiles (DM, M) qui peuvent initier la formation d'une autre génération de schizontes dans d'autres cellules intestinales (2-5) ou deviennent des gamontes de différents sexes. Les microgamontes (7) plus rares ont un noyau qui se divise de nombreuses fois donnant des microgamontes multinucléaires (7.1) qui engendrent plusieurs microgamètes flagellés (7.2). Les gamontes femelles unicellulaires (8) les plus nombreux, grossissent et ne se divisent pas, chacun devenant un macrogamète femelle (8.1) qui se caractérise par l'apparition de deux types d'éléments constitutifs de la membrane $\left(\mathrm{WF}_{1}, \quad \mathrm{WF}_{2}\right)$. Après la fertilisation, le nouveau zygote (9) forme la coque de l'oocyste à partir de la fusion consécutive des deux types de précurseurs de membrane (FW). Les oocystes non sporulés (10) sont excrétés par les fèces (à l'exception des espèces de Eimeria parasitant les reptiles et les poissons).

\section{Développement exogène}

Le cycle débute par l'élimination des oocystes (11) dans le milieu extérieur avec les excréments. Si les conditions suivantes sont réunies à savoir:

- une humidité de l'ordre de $70 \%$

- une température de l'ordre de $26^{\circ}$ à $30{ }^{\circ} \mathrm{C}$ 
- une oxygénation convenable,

l'oocyste va alors évoluer et donner 4 cellules non différenciées appelées sporoblastes (12). L'évolution aboutit à un oocyste sporulé (13) contenant 4 sporocystes contenant chacun 2 sporozoïtes. $\mathrm{Ce}$ développement exogène est encore appelé sporogonie. Il a lieu dans la litière et dure 24 à 48 heures. En effet, le sporonte diploïde subit en 1-2 jours à l'extérieur de l'hôte une sporogonie avec la réduction chromatique produisant 4 sporoblastes haploïdes qui s'enkystent, formant des sporocystes. Dans chaque sporocyste, une division donne 2 sporozoïtes.

\section{Pathogenèse}

Le pouvoir pathogène des coccidies varie selon l'espèce de coccidie en cause, le nombre d'oocystes ingérés et la compétence immunitaire de l'oiseau hôte. L'espèce la plus pathogène est $E$. tenella suivi de E. necatrix, E. brunetti et $E$. acervulina avec la mortalité des sujets affectés à dose élevée. Selon les travaux de William (2001), la dose létale est de 18.200, 63.000 et 16.300 oocystes pour Eimeria brunetti, Eimeria necatrix et Eimeria tenella, respectivement. La mortalité peut atteindre $100 \%$ à partir d'une dose de 1000000 d'oocystes au niveau des espèces Eimeria tenella et Eimeria necatrix. Les autres espèces de coccidies ne causent généralement pas de mortalité, mais réduisent significativement les performances de croissance et de ponte avec une incidence économique remarquable. Les diverses espèces de coccidies et les lésions qu'elles engendrent sont consignées dans le Tableau 1.

\section{Diagnostic de la coccidiose}

La coccidiose se présente souvent sous les deux formes suivantes : la forme clinique avec la manifestation des signes cliniques de la maladie et la forme asymptomatique à effets désastreux sur les performances de production. Le caractère silencieux de certaines coccidioses rend le diagnostic parfois très difficile. Les méthodes parasitologiques empiriques utilisées sont laborieuses. Des caractéristiques d'ordre pathologique permettent de faire une tentative d'identification des espèces, ce sont : le site ou la localisation des lésions dans l'intestin, l'aspect des lésions macroscopiques, La dimension, la forme et la couleur des oocystes, la dimension des schizontes et des mérozoïtes, le type de tissu parasité, la période prépatente minimale dans une infection expérimentale, le temps minimal de sporulation et l'immunogénécité contre un stérotype connu (McDougald, 2003).

L'identification des oocystes coccidiens et leur comptage ne permettent pas réellement de mesurer l'ampleur de la maladie. Williams (2001) a démontré qu'un organisme infecté plus résistant à la coccidiose est capable de produire plus d'oocystes qu'un organisme infecté plus sensible. Le score de lésions dans le diagnostic permet d'appréhender l'importance de la maladie basée sur l'appréciation des lésions macroscopiques suivant la méthode mise au point par Johnson et Reid (1970). Le score $0=$ normal et le score $4=$ coccidiose sévère. Une lésion dont le score est inférieur ou égal à 1,5 est associée à une coccidiose sub-clinique et une lésion dont le score est supérieur à 1,5 à une coccidiose clinique. Le score est réalisable sur des lésions causées par des espèces plus pathogéniques des coccidies telles que E. acervulina et E. tenella. Le score de lésion dans le diagnostic devient inefficace au niveau des espèces comme: E. mitis et $E$. praecox qui causent une coccidiose silencieuse subclinique mais ayant une incidence économique remarquable (Gore et long, 1982). La méthode ancienne 
d'identification des espèces de coccidie utilise la théorie de l'immunité spécifique caractéristique du genre Eimeria avec des infections expérimentales répétées de souche pure (McDougald, 2003). La localisation des lésions dans le tractus gastro-intestinal à la nécropsie et la morphométrie au microscope photonique permettent d'identifier les espèces.

De récentes méthodes biochimiques et moléculaires par le PCR (Polymerase Chain Reaction) de plus en plus précises permettent d'identifier les espèces de coccidies à partir du génome du parasite (Morris et Gasser, 2006; Vancraeynest et al., 2011). Cette technique constitue une avancée notoire scientifique en matière de diagnostic, mais le coût élevé et son aspect uniquement qualitatif limite son utilisation. Une méthode novatrice d'identification des espèces de coccidies des poulets et celles des lapins est mise au point par Gruber et al. (2007). Il s'agit d'une morphométrie informatisée qui utilise les images numérisées des espèces de coccidies confirmées comme une référence d'identification à l'aide d'un logiciel dénommé COCCIMORPH. Le logiciel compare l'image de la coccidie à identifier à la référence existant dans le programme et attribue un pourcentage de ressemblance. Les règles d'utilisation exigent que l'image de coccidies à identifier soit d'une résolution très élevée, prise avec un appareil photo de plus de 4 mégapixel avec un grossissement de 40x au microscope photonique. L'avantage de cette méthode est son accessibilité facile et son coût faible. Toutefois, seuls les oocystes sporulés sont identifiables.

\section{Prévention et contrôle de la coccidiose}

La lutte contre la coccidiose est basée sur la prévention médicamenteuse ou vaccinale et le traitement à la suite d'un diagnostic.

\section{Prévention}

Bonne gestion et l'hygiène de la litière, l'utilisation des anticoccidiens dans la ration alimentaire des oiseaux et l'utilisation des vaccins sont les mesures de biosécurité mises en place pour la prévention de la coccidiose.

\section{Gestion de la litière}

Comme les oocystes coccidiens sont omniprésents et facilement disséminés partout où se trouve une unité avicole, il devient très difficile de maintenir les poulets exemptes de coccidies. Les oocystes sporulent très facilement dans la litière des poulaillers. Pourtant, la présence d'autres bactéries, microorganismes et de l'ammoniac peut déranger leur survie, entraînant la diminution de leur viabilité après 3 semaines (Williams, 1995). La pratique courante du renouvellement de la litière et de réalisation du nettoyage systématique des locaux d'élevage avant la réception d'une nouvelle bande d'oiseaux favorise une bonne aération, réduit considérablement la charge parasitaire coccidienne et minimise la dissémination des oocystes infectieux. Ces dispositions de biosécurité se répandent de plus en plus dans la mesure où l'on assiste à une dégradation continue de l'efficacité des anticoccidiens et une augmentation de l'utilisation des vaccins.

\section{Médicaments anticoccidiens de prévention}

Des médicaments utilisés contre les coccidioses de poulets sont des deux catégories suivantes: les anticoccidiens antibiotiques ionophores et les anticoccidiens synthétiques. La prévention par l'utilisation des médicaments anticoccidiens dans la ration alimentaire ou chimio-prophylaxie occupe $95 \%$ des méthodes de prévention (De Gussem, 2005). Les médicaments présentés dans le Tableau 2 sont ceux autorisés aux EtatsUnis d'Amérique (Miller, 1989).

En Europe, les anticoccidiens suivants sont autorisés chez le poulet de chair 
selon Vancraeynest et al. (2011), quatre sont des composés chimiques (Robénidine, Diclazurile, Nicarbazine et Décoquinate) et 6 sont des ionophores (les ionophores monovalents: Salinomycine, Monensin et Narasin; les ionoplores glycosides monovalents: Maduramycine et Senduramycine et les ionophores bivalents: Lasalocide). En dehors des médicaments, certains additifs alimentaires tels que les sources de matières grasses contenant une forte concentration des acides gras n-3 (AG n-3) (acide docosahexanoique, acide eicosapentanoique et acide linoleique), provenant des huiles de poisson, réduisent considérablement les lésions résultant de l'infection expérimentale des poussins à Eimeria tenella (Allen et al., 1998).

\section{Vaccination}

\section{Immunobiologie de la coccidiose}

Les poussins d'un jour ne tirent pas une immunité protectrice passive de la mère poule et les oiseaux de tout âge sont donc sensibles à la coccidiose. En pratique, les oiseaux sont infectés dès les premières semaines et cette infection induit une bonne immunité qui persiste toute la vie de l'oiseau et est entretenue par des infections coccidiennes mineures fréquentes. Cette immunité peut décliner en l'absence d'infection. Un aspect cardinal de cette immunité est sa spécificité à l'espèce de coccidie. Ainsi, chez les poulets par exemple, l'immunité contre Eimeria tenella ne confère pas une résistance contre Eimeria maxima (McDougald, 2003). A l'intérieur d'une même espèce, très peu de variation sérotypique existe remarquablement, car des espèces isolées dans des zones agro-écologiques différentes peuvent induire des protections croisées substantielles. Néanmoins, Eimeria acervulina constitue une exception importante, car l'immunité induite par cette espèce nécessite des expositions répétées à des doses faibles d'oocystes appelées infections à compte goutte. Des travaux en infection expérimentale ont montré que l'immunité est totalement acquise et installée après trois contacts avec toutes les espèces de coccidies du poulet et même parfois en un cycle avec certaines espèces comme E. maxima, le cycle durant une semaine et l'immunité 3 semaines (Bostvironnois et Zadjian, 2011). Dans les conditions naturelles d'élevage, l'immunité peut être établie en 3 semaines d'âge ou plus tard en 6 ou 7 semaines (Chapman, 1999). Elle se manifeste par une réduction sensible des lésions et de production d'oocystes dues non seulement à la réduction du nombre de sporozoïtes qui avec succès envahissent la cellule hôte, mais aussi à l'inhibition de leur développement intracellulaire (McDougald, 1997). Les inducteurs de réaction immunitaire à une infection coccidienne primaire, sont en premier lieu les cellules $\mathrm{T}$ situées dans les tissus lymphoïdes de la lumière intestinale (Chapman, 1999). La réponse immunitaire humorale ( $\operatorname{IgA})$ également a eu lieu mais les anticorps jouent un rôle mineur dans la résistance et l'immunité contre les coccidies selon le même auteur. Les rapports récents de Leinsing (2009) ont montré que les améliorations génétiques ces dernières années ont considérablement affaibli l'immunité des oiseaux.

\section{Vaccins}

Une bonne immunité vaccinale est conférée aux oiseaux par des vaccins vivants à virulence modérée (Chapman et al., 2005). Les vaccins tués ont un cycle de vie détérioré surtout pendant la phase asexuée, ce qui réduit considérablement le potentiel reproductif et pathogénique caractéristique des bons vaccins. Les vaccins vivants, malgré leur pouvoir pathogène, constituent un arsenal essentiel dans l'efficacité des anticoccidiens utilisés 
dans l'industrie avicole. Il est bien établi de nos jours que même les espèces les plus immunogènes comme Eimeria maxima ont besoin d'une réinfection à 8 semaines d'âge du poussin après une primovaccination le jour d'éclosion (Chapman et al., 2005). De nouvelles méthodes de vaccinations ont été développées mais l'efficacité des différentes voies d'administration et la couverture vaccinale restent à élucider.

Par ailleurs, on distingue deux catégories de vaccins vivants: les vaccins tolérants et les vaccins sensibles aux médicaments anticoccidiens ionophores. Les vaccins tolérants aux ionophores ont pour avantage majeur de permettre l'utilisation des ionophores pendant les 3 à 4 premières semaines post vaccinales où l'immunité n'est pas encore complète et l'oiseau demeure toujours vulnérable (Chapman et al., 2005). Les anticoccidiens limitent la pression des infections coccidiennes du milieu pendant la période de développement de l'immunité (Vermeulen et al., 2001). Les vaccins tolérants aux produits anticoccidiens également peuvent altérer le niveau de résistance d'une certaine population de coccidies (Mathis, 2003 ; Chapman et al., 2004 ; Mathis et Broussard, 2006 ; Peek et Landman, 2006). Cette approche, basée sur l'association des anticoccidiens aux vaccins vivants tolérants à ces anticoccidiens, est fortement utilisée pour optimiser l'efficacité des anticoccidiens par la réduction de la résistance d'une portion des parasites (Chapman et McFarland, 2003). Des programmes de rotation entre les anticoccidiens et les vaccins vivants se substituent valablement à la gestion traditionnelle de contrôle des coccidies basée sur la rotation entre deux ionophores ou un ionophore et un anticoccidien synthétique.
L'amélioration de l'utilisation de la compétence immunitaire des oiseaux pour le contrôle de la coccidiose a conduit à la mise au point des vaccins recombinés sur la base des sérotypes vaccinaux et des molécules spécifiques du parasite. Plusieurs molécules des Apicomplexa (Micronemes) ont prouvé leur utilité prophylactique contre les coccidies (Tewari et al., 2010). Des protéines micronemes de E. tenella comme antigène (Etmic2 et Etmic4) ont démontré un effet stimulateur d'immunité considérable (Du et Wang, 2005). Des protéines associées au stage sexuel du cycle de E. maxima (kDA) interviennent dans la protection immunitaire des sujets expérimentalement infectés (Lee et al., 2009). Ces protéines gamétocytaires induisent une immunité intestinale protective qui déprime la production d'oocystes et réduit les lésions intestinales (Jang et al., 2010) avec l'amélioration des performances de croissance (Anwar et al., 2008). Les cytokines (interféron gamma) constituent les produits résultant de la réponse immunitaire cellulaire vaccinale et de ce fait, sont de plus en plus associées aux vaccins comme adjuvants, ce qui améliore considérablement l'immunité (Lillehoj et al., 2000). Berezin et al. (2010) ont démontré l'efficacité du vaccin constitué d'un antigène de E. tenella et d'un Complexe Immuno-stimulateur (saponine). Toutefois, les vaccins vivants, quelle que soit la virulence, doivent se répliquer dans les cellules hôtes épithéliales pour conférer à l'organisme une immunité active. Cette phase de multiplication entraîne un état morbide coccidien sub-clinique avec des effets majeurs de baisse de performance et de prédisposition du tractus intestinal à des infections bactériennes gastro-intestinales opportunistes avec de l'entérite, les fientes humides, une augmentation des lésions podales et de l'indice de consommation (Bostvironnois et Zadjian, 2011). 
Mode d'action, résistance des coccidies et toxicité des anticoccidiens

Mode d'action des anticoccidiens

Plusieurs stratégies sont adoptées en matière d'utilisation des médicaments. L'utilisation d'un seul médicament du premier jour jusqu'à l'abattage de la bande est pratiquée par certains éleveurs (McDougald, 2003). D'autres aviculteurs préfèrent la méthode de rotation qui permet d'utiliser plusieurs médicaments d'une manière cyclique afin de réduire les risques de résistance des coccidies aux médicaments (Chapman et al., 2005)

Les coccidies sont sujettes à l'attaque des médicaments à des stages variés de leur développement, à l'intérieur de l'hôte. Des médicaments de principes actifs totalement différents peuvent agir sur la même étape de développement du parasite. Selon Jeffers (1997), l'Amprolium rivalise avec l'absorption de la thiamine par le parasite, tandis que Les Quinolones et les Clodipoles arrêtent les parasites par l'inhibition du métabolisme d'énergie dans le cytoplasme. D'autres principes actifs comme les Ionophores tuent les sporozoïtes ou les nouvelles trophozoïtes. Les Nicarbazines, Robénidines et les Zoalenes détruisent les schizontes de première et deuxième génération et les Sulfonamides avec les médicaments dérivés font de la compétition pour l'incorporation de l'acide folique. Ils agissent sur les schizontes en développement et les phases de divisions sexuées. Les Diclarzuriles agissent sur la première schizogonie avec Eimeria tenella mais sur la dernière schizogonie avec Eimeria acervulina et sur les macrogamètes en maturité chez Eimeria maxima (Chapman, 1999).

\section{Résistance des coccidies aux anticoccidiens}

Des degrés de résistances aux anticoccidiens y compris aux Ionophores ont été développés et l'utilisation continue et routinière des anticoccidiens a entraîné l'émergence des sérotypes résistants aux médicaments (Long, 1982). Toutefois, certains médicaments continuent d'être utilisés par l'industrie avicole de nos jours comme l'Amprolium, Nicarbazine, Robénidine, Diclarzurile, Zoalene, décoquinate et Halofuginone. Ainsi, leur utilisation est la preuve de leur potentiel faible d'induction de résistance au niveau des parasites, comparé à d'autres produits qui ont certainement disparu (De Gussem, 2005). La résistance des coccidies à un médicament est évaluée par la procédure ASTs (Anticoccidial Sensitivity Tests) (McDougald et al., 1987; Peek et Landman, 2003; Naciri et al., 2003). C'est un anti-coccigramme qui permet de tester in vivo l'efficacité d'un anticoccidien sur des coccidies de souche connue. Pour minimiser l'effet de ces phénomènes de résistance, certains aviculteurs font une rotation d'utilisation des divers anticoccidiens sur des bandes successives. Ils combinent aussi les autres composées chimiques avec les Ionophores ou emploient des programmes dualistes, qui consistent à utiliser plusieurs produits anticoccidiens au cours du développement d'une bande de volaille. Toutefois, l'application de ce programme, dépend de la saison et de la prévalence des diverses espèces de coccidies (William, 1998). Les compagnies pharmaceutiques fabriquent deux sortes de médicaments qui ciblent dans leur action, l'enzyme du cycle de manitole de la sporozoïte et de la histone diacetylase de la trophozoïte (Schmatz, 1997; Allocco et al., 1999).

\section{Effet des anticoccidiens sur les poulets}

La plupart des anticoccidiens ont une toxicité sélective active sur les parasites et non toxique pour les vertébrés en général. Malheureusement, certains médicaments peuvent posséder des effets secondaires nocifs pour les oiseaux. Le surdosage des anticoccidiens dans certains cas peut constituer une source de toxicité. Des interactions de principes actifs médicamenteux et de certains facteurs tels 
que: le mode de gestion, le profile génétique des oiseaux et leur état nutritionnel, peuvent constituer des sources majeures de toxicité chez les oiseaux (McDougald, 2003). Il s'agit:

- du Nicarbazine, déjà reconnu toxique aux poules pondeuses avec la décoloration de la coquille marron des œufs et le jaune d'œuf marbré; ce qui réduit considérablement le taux d'éclosion. Son interaction avec un environnement caractérisé par une température élevée peut se révéler toxique aux oiseaux avec une forte mortalité;

- des Ionophores qui, à dose élevée, sont généralement toxiques provoquant la dépression du gain de poids vif corporel, une paralysie transitoire ou permanente et la mortalité;

- du Monensin qui peut agir sur la méthionine pour réduire la poussée des plumes. Les Lasalocides toxiques stimulent une consommation d'eau excessive et entraîne l'humidité de la litière.

\section{Plantes médicinales dans la lutte contre la coccidiose}

L'utilisation des antioxidants comme supplément alimentaire tels que les tocophéroles $(8 \mathrm{ppm})$ retrouvés dans les huiles végétales, le blé, le maïs, les graines de soja et certaines composées médicinales (curcumine, 0,05\%) apparaît efficace contre la coccidiose due à $E$. acervulina et E. maxima (Allen et al., 1998). L'artemisine, une herbe chinoise, extraite de Artemisia annua qui détient une propriété antipaludique (endoperoxide), est aussi efficace sur les coccidioses dues à E. acervulina et E. tenella par la réduction de la production d'oocystes (Allen et al., 1997). L'incorporation de la poudre des feuilles séchées de Andrographis paniculata à 10\%, 20\%, $30 \%$ et $40 \%$ dans l'aliment a eu un effet positif sur la mortalité dont $0 \%$ et $42,85 \%$ pour le groupe alimenté avec $40 \%$ de
Andrographis et le groupe témoin respectivement (Sujikara, 2000). Des quinze plantes médicinales asiatiques telles que: Gleditsia japonica, Melia azedarach, Torilis japonica, Artemisia annua, Artemisia asiatica, Quisqualis indica, Bupleurum chinese, Inula helenium, Sophora flavescens, Sophora japonica, Torreya nucifera, Ulmus macrocarpa, Sinomenium acutum, $P$. Koreana, $P$. aviculare, testées contre la coccidiose due à E. tenella, seule Sophora flavescens a été la plus efficace sur la réduction de la diarrhée sanguinolente, le score de lésion, la production d'oocystes et l'amélioration du gain de poids (Youn et Noh, 2001). Les extraits de Allium sativum, Salvia officinalis, Echinacea purpurea, Thymus vulgaris et Origanum vulgare testés sur les coccidioses dues à $E$. tenella, $E$. acervulina, E. maxima et E. necatrix, ont donné des résultats similaires à ceux du coccidiostatique conventionnel utilisé sur le lot témoin infecté et traité de la souche Ross 308 en termes de gain de poids vif corporel et de production d'oocystes (Arczewska et Swiatkiewicz, 2010).

\section{Resistance naturelle dans la lutte contre la coccidiose}

\section{Définition de la résistance naturelle}

Certaines particularités de la filière avicole, comme les fortes densités d'élevage augmentant le risque de transmission, l'homogénéité génétique des populations empêchant le rôle de barrière de génotypes plus résistants ou encore la qualité sanitaire des troupeaux évitant toute sélection naturelle, favorisent la propagation des agents pathogènes (Caleng et al., 2011). La coccidiose est une pathologie d'intérêt majeur à incidence économique remarquable, tant sur le plan coût du traitement conventionnel que l'aspect sub-clinique silencieux de la maladie. Elle constitue alors l'une des maladies cibles dans les programmes de sélection génétique visant l'utilisation de la résistance naturelle des 
oiseaux pour son contrôle (Davies et al., 2009).

La résistance génétique aux maladies est la prédisposition naturelle d'un sujet à faire face avec succès aux effets adverses des agents pathogènes. Les deux sortes de résistance suivantes sont distinguées: la résistance vraie ou résistance à l'infection, quand le génotype hôte évite l'invasion par le pathogène, et la résistance partielle ou résilience, quand le pathogène envahit l'animal mais sans causer de maladie sévère (Bishop et Wooliams, 2010). Il existe également la résistance trans-espèce hôte, celle qui peut exister entre les végétaux et les animaux par exemple (Gavora, 1990). De même, peut être considéré la résistance ou portage, qui désigne la capacité des animaux à éliminer rapidement le pathogène de leur organisme (Calenge et al., 2011). La prise en compte de ce critère est primordiale pour limiter le potentiel de dissémination du germe pathogène, sa transmission à d'autres animaux et son maintien dans l'environnement de production.

Variation de la sensibilité à la coccidiose Déjà Rosenberg (1954) a obtenu un taux de divergence de $35 \%$ de sensibilité entre deux souches de poulet en réponse à l'infection avec l'espèce Eimeria tenella. Long (1968) et Bumstead et Millard (1987) ont exposé différentes souches de poulet à plusieurs espèces de Eimeria et ont mesuré la résistance à travers le gain de poids vif corporel, la mortalité et l'excrétion d'oocystes. L'influence de la souche hôte sur l'expression de la maladie fut clairement démontrée. Après 12 générations de sélection par rapport à la sensibilité à Eimeria tenella, les mortalités pour le groupe témoin, le groupe résistant, le groupe peu résistant et le groupe plus sensible, étaient respectivement $41 \%, 21 \%$, $29 \%$ et $35 \%$ (Johnson et Edgar, 1982). Pinard et al. (1998) ont trouvé la souche Egyptienne Fayoumi plus résistante des 5 autres testées, basée sur la mortalité, le score de lésions et la réduction de croissance. De récentes études toujours sur le génotype Fayoumi ont montré que la souche Fayoumi M5.1, a été plus résistante à la coccidiose que la souche Fayoumi M15.2 (Kim et al., 2009). Ayissiwedé et al. (2011) ont montré que le poulet local sénégalais est plus résistant à la coccidiose que les souches exotiques, la Cobb 500 et la Hy-line W-36. Au Bénin, Dakpogan et al. (2012) ont observé une différence de sensibilité à la coccidiose due à Eimeria tenella au sein de la population du poulet local avec, le phénotype cou nu qui s'était révélé plus tolérant à la coccidiose surtout en termes de production différentielle d'oocystes.

\section{Cause de la variation de la sensibilité à la coccidiose}

Tyzzer et al. (1932) ont démontré que la production divergente d'oocystes de Eimeria tenella entre plusieurs sujets est due à la différence de disponibilité initiale des hôtes potentiels que représentent des cellules épithéliales caecales et la mutation de l'épithélium caecal au cours de l'infection avec la formation des noyaux caecaux qui empêchent des décharges de mérozoïtes. La compétence immunitaire individuelle est fortement impliquée dans la variation de la sensibilité à la coccidiose. L'altération de la population des lymphocytes et celle de la production de la cytokine pendant le cours de l'infection coccidienne à été investiguée pour clarifier la nature de la protection immunitaire des oiseaux (Bessay et al., 1996). Les résultats ont démontré que l'interféron gamma est un composant important de l'immunité cellulaire protective de l'hôte. D'autres auteurs ont montré que la réponse immunitaire cellulaire de différents sujets (Ovington et al., 1995), avec la réaction lymphoproliférative $\left(\mathrm{CD}^{4+} / \mathrm{CD}^{8+}\right)$ (Talebi et Mulcahy, 1995) et la sécrétion d'interféron 
gamma (Del Cacho et al., 2011), interviennent dans la différence de sensibilité entre plusieurs souches de poulets. Les expériences conduites par Muhammad et al. (2010) sur plusieurs variétés de poulet ont mis en évidence l'effet inhibiteur de l'interféron gamma, une cytokine majeure, sur la prolifération de Eimeria tenella. Kim et al. (2009) ont suggéré l'existence d'un déterminant génétique au niveau du Complexe Majeur d'Histocompatibilité (CMH) qui influence la sensibilité des oiseaux à la coccidiose en contrôlant l'expression locale et systémique de la molécule de cytokine et de la kémokine. Gavora (1990) a montré l'évidence de la corrélation entre les critères de mesure de résistance aux espèces de Eimeria et les haplotypes du Complexe Majeur d'Histocompatibilité $(\mathrm{CMH})$. Les haplotypes $\mathrm{B}^{5}$ et le $\mathrm{B}^{15}$ apportent plus de protection contre Eimeria tenella et Eimeria acervulina que les $\mathrm{B}^{2}$, $\mathrm{B}^{12}$ ou $\mathrm{B}^{13}$. Des différences considérables en résistance ont été observées entre des souches qui partagent les mêmes allèles $\mathrm{CMH}$ (ex. $\mathrm{B}^{13}$ ) mais ayant différentes origines génétiques (Lillehoj et al., 1989); résultats corroborés par les travaux de Ruff et Bacon (1989) et Dunnington et al. (1992). L'implication de loci en dehors de CMH dans la détermination de la résistance à Eimeria a été rapporté (Johnson et Edgar, 1984; Martin et al., 1986).

Tableau 1: Signes et lésions des espèces coccidiennes les plus pathogènes du poulet.

\begin{tabular}{llllll}
\hline Espèces & Sites & Lésions macroscopiques & MPP & STM & Signes \\
\hline E. brunetti & $\begin{array}{l}\text { Partie } \\
\text { postérieure de } \\
\text { l'intestin }\end{array}$ & $\begin{array}{l}\text { Coagulations nécrotiques, } \\
\text { entérite mucoïde et } \\
\text { hémorragique }\end{array}$ & 120 & 18 & $\begin{array}{l}\text { Diarhhée } \\
\text { sanguinolente }\end{array}$ \\
\hline E. necatrix & Intestin & $\begin{array}{l}\text { Ballonnement, spots } \\
\text { blanchâtres, hémorragie } \\
\text { pétéchiale, exudation } \\
\text { mucoïde et sanguinolente }\end{array}$ & 138 & 18 & Déshydration \\
\hline E. tenella & Caeca & $\begin{array}{l}\text { Hémorragie dans la } \\
\text { lumière, muqueuse épaisse } \\
\text { et blanchâtre, noyaux de } \\
\text { sang coagulé }\end{array}$ & 115 & 18 & Anémie \\
\hline $\begin{array}{l}\text { E. } \\
\text { acervilina }\end{array}$ & $\begin{array}{l}\text { Partie } \\
\text { postérieure de } \\
\text { l'intestin }\end{array}$ & $\begin{array}{l}\text { Lésions rondes et } \\
\text { blanchâtre (Infection } \\
\text { légère), plaies coalescentes } \\
\text { membrane intestinale } \\
\text { épaisse (infection lourde) }\end{array}$ & 97 & 17 & $\begin{array}{l}\text { Réduction de } \\
\text { croissance }\end{array}$ \\
\hline E. maxima & Intestin & $\begin{array}{l}\text { Membrane intestinale } \\
\text { épaisse, exudation } \\
\text { mucoïde, hémorragie } \\
\text { pétéchiale }\end{array}$ & 121 & 30 & $\begin{array}{l}\text { Consommatio } \\
\text { d'aliment et } \\
\text { d'eau }\end{array}$ \\
\hline
\end{tabular}

TMS: Temps minimal de sporulation, PPM: Période prépatente minimale, Source : Modifié de McDougald (2003). 
Tableau 2: Médicaments anticoccidiens utilisés à titre préventif.

\begin{tabular}{lcc}
\hline Médicaments & AAM: 1 $^{\text {ere }}$ Approbation & Délai d'élimination \\
\hline Sulfaquinoxaline & 1948 & 10 \\
Nitrofurazone & 1948 & 5 \\
Arsanilic acide ou sodium & 1949 & 5 \\
arsanilate & & \\
Nicarbazine & 1955 & 4 \\
Furazolidone & 1957 & 5 \\
Oxytetracycline & 1959 & 3 \\
Amprolium & 1960 & 0 \\
Chlortetracycline & 1960 & \\
Zoalene & 1960 & 5 \\
Buquinolate & 1967 & 0 \\
Clodipole ou meticlorpindole & 1968 & 5 \\
Decoquinate & 1970 & 0 \\
Sulfadimethoxine, ormetoprine & 1970 & 5 \\
Monensin & 1971 & 0 \\
Robenidine & 1972 & 5 \\
Lasalocide & 1976 & 3 \\
Salinomycine & 1983 & 0 \\
Halofuginone & 1987 & 5 \\
Narasin & 1988 & 0 \\
Madurimicine & 1989 & 5 \\
Narasin + nicarbazine & 1989 & 5 \\
Semduramycine & 1995 & 0 \\
\hline
\end{tabular}

AAM: Administration d'Aliment et de Médicament ; Source : Modifié de Miller (1989)

Tableau 3: Médicaments utilisés en traitement aux Etat-Unis.

\begin{tabular}{|c|c|c|c|}
\hline Médicaments & Aliment ou eau & AAM: $1^{\text {ère }}$ Approbation & $\begin{array}{c}\text { Délai d'élimination } \\
\text { (Jours) }\end{array}$ \\
\hline Sulfamethazine & Eau & 1947 & 10 \\
\hline Sulfaquinoxaline & Aliment & 1948 & 10 \\
\hline Amprolium & Eau & 1960 & 0 \\
\hline $\begin{array}{l}\text { Sulfachloropyrazine } \\
\text { monohydraté }\end{array}$ & Eau & 1967 & 4 \\
\hline Sulfadimethoxine & $\mathrm{Eau}$ & 1968 & 5 \\
\hline
\end{tabular}

AAM : Administration d'Aliment et de Médicament ; Source : Miller (1989)

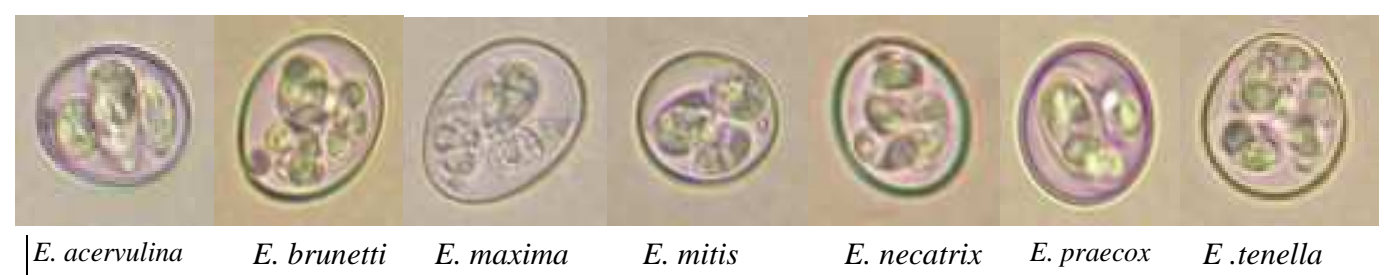

Figure 1: Eimeria spp. du poulet (Gruber et al., 2007). 


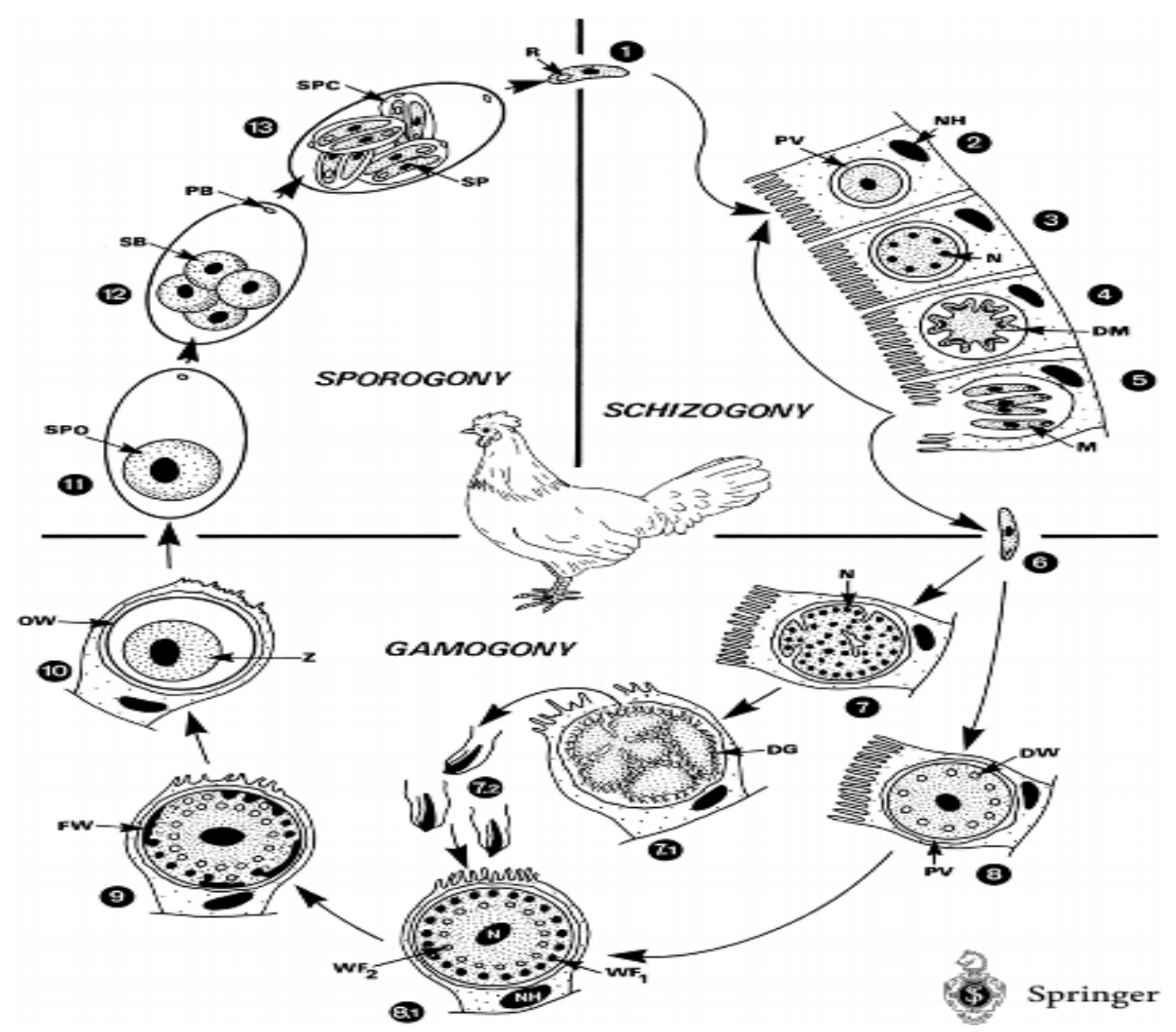

DG = microgamète en développement, $\mathbf{D M}=$ mérozoïte en développement, $\mathbf{D W}=$ précurseur de la coque, $\mathbf{F W}=$ fusion de la WF1 pour former la couche externe de la $\mathbf{O W}, \mathbf{M}=$ mérozoïte, $\mathbf{N}=$ noyau, $\mathbf{N H}=$ noyau de la cellule hôte, $\mathbf{O W}=$ la coque de l'oocyste, $\mathbf{P B}=$ corps polaire (granule), $\mathbf{P V}=$ vacuole parasitophore, $\mathbf{R}=$ corps refractile ou de réserve, $\mathbf{S B}=$ sporoblaste, $\mathbf{S P}=$ sporozoïte, $\mathbf{S P C}=$ sporocyste, $\mathbf{S P O}=$ sporonte, $\mathbf{W F 1}=$ corps précurseur 1 de la coque, $\mathbf{W F 2}=$ corps précurseur 2 de la coque, $\mathbf{Z}=$ zygote ou jeune oocyste.

Figure 2: Cycle biologique des coccidies du poulet (McDougald, 2003).

\section{Conclusion}

Le contrôle de la coccidiose a commencé depuis la découverte de la sulfanilamide par Levine (1939). Les produits variés en l'occurrence les Ionophores ont été intensément utilisés pour prévenir et guérir la coccidiose chez le poulet. Les problèmes de résistances des coccidies aux anticoccidiens et la présence de résidus médicamenteux dans les produits et sous-produits de la volaille sont des préoccupations majeures. Les limites de cette chimiothérapie ont entraîné le développement des vaccins vivants et plus récemment des vaccins recombinés. La biosécurité couramment pratiquée consiste à associer aux vaccins, des médicaments auxquels ils sont tolérants dans l'ultime but d'améliorer l'efficacité des deux produits. Malgré les nombreuses preuves de l'efficacité de certaines plantes médicinales sur les coccidies, les produits utilisés en prévention ou en traitement contre la coccidiose n'ont pas varié. La 
coccidiose sub-clinique est une menace certaine pour l'aviculture car malgré les mesures de biosécurité anticoccidiennes mises en œuvre, les coccidies continuent d'être directement ou indirectement les causes des entérites, et des lésions podales consécutives à la litière humide. Les vaccins vivants utilisés, en eux-mêmes, au cours de leur multiplication dans les entérocytes, sont des sources d'infection sub-clinique à effet néfaste sur les performances de production des poulets. Les vaccins vivants constituent également un facteur prédisposant pour les bactéries opportunistes en l'occurrence Clostridium perfringens. La sélection de gène de production a été tout le temps, le point focal des investigations en termes d'amélioration des performances. La recherche des gènes majeurs de résistance naturelle des poulets à la coccidiose dans les programmes de sélection en aviculture doit permettre d'éradiquer les pertes énormes engendrées par le coût élevé des mesures de contrôle et de réduire considérablement et durablement l'incidence économique consécutive à la baisse des performances de poulets due à la coccidiose silencieuse sub-clinique.

\section{REFERENCES}

Allen PC, Danforth HD, Levander OA. 1997. Interaction of dietary flaxseed with coccidia infections in chickens. Poult Sci., 76: 822-827.

Allen PC, Danforth HD, Augustine PC. 1998. Dietary modulation of aviancoccidiosis. Int. Parasitol., 28: 1131-1140.

Allocco JJ, Profous-Juchelka H, Myers RW, Nare B, Schmatz DM. 1999. Biosynthesis and catabolism of mannitol is developmentally regulated in the protozoan parasite Eimeria tenella. J. Parasitol., 85: 167-173.

Anwar MI, Akhtar M, Hussain I, Muhammad F, Haq AU. 2008. Effects of local gametocyte and livacox vaccines on live body weight gain and lymphoid organs in chickens. Pakistan. Vet. J., 3: 136-138.

Arczewska-Wlosek A, Swiatkiewicz S. 2010. Response of Chickens Infected With Coccidiosis to Herbal Extracts Mix Fed Singly or In Combination with Additives. XIIIth European Poultry Conference, p. 45-50.

Ayssiwede SB, N'dri KM, Gbati O, Missohou A. 2011. Etude comparée de la sensibilité de différentes souches de poules à la coccidiose aviaire. Revue Méd. Vét., 162(3): 138-142.

Berezin VE, Bogoyavlenskyi AP, Khudiakova SS, Alexuk PG, Omirtaeva ES, Zaitceva IA, Tustikbaeva GB, Barfield RC, Fetterer RH. 2010. Immunostimulatory complexes containing Eimeria tenella antigens and low toxicity plant saponins induce antibody response and provide protection from challenge in broiler chickens. Vet. Parasitol., 167(1): 28-35.

Bessay M, Le Vern Y, Kerboeuf D, Yvore P, Quere P. 1996. Changes in intestinal intra-epithelial and systemic T-cell subpopulations after an Eimeria infection in chickens: comparative study between $E$. acervulina and $E$. tenella. Vet. Res., 27: 503-514.

Bishop S, Wooliams J. 2010. On the genetic interpretation of disease data. PloS ONE, 5(1): e 8940.

Bostvironnois C, Zadjian C. 2011. Coccidiose subcliniques en production de poulet de chair: Bilan et prospectives. Neuvième Journée de Recherche Avicole, Tours, p. 585-588.

Bumstead N, Millard BJ. 1987. Genetics of resistance to coccidiosis: Response of inbred chicken lines to infection by Eimeria tenella and Eimeria maxima. Br. Poult. Sci., 28: 705-716.

Caleng F, Pinard-van der Laan MH, Beaumont C. 2011. Apport de la génomique à l'étude de résistance génétique aux maladies. Neuvième 
Journée de Recherche Avicole, Tours, p. $45-461$.

Cannavan A, Ball G, Kennedy DG. 2000. Nicarbazine contamination in feeds as a cause of residue in eggs. Food Additive Contamination, 25: 829-836.

Chapman HD. 1999. The development of immunity to Eimeria species in broilers given anticoccidial drugs. Avian Pathol., 28: 155-162.

Chapman HD, McFarland JL. 2003. Rotation programs with Diclazuril and a coccidiosis vaccine. Proc. $52^{\text {nd }}$ WPDC, Sacramento.

Chapman HD, Matsler P, LaVorgna MW. 2004. The effects of salinomycin and roxarsone upon the performance of broilers when included in the feed for four, five, or six weeks and infected with Eimeria species during the starter or grower phase of production. Poult. Sci., 83: 761-764.

Chapman HD, Matsler PL, Muthavarapu VK, Chapman ME. 2005. Acquisition of immunity to Eimeria maxima in newly hatched chickens given 100 oocysts. Avian Disease, 49(3): 426429.

Danaher M, Campbell K, O'Keeffe M, Capurro E, Kennedy G, Elliott CT. 2008. Survey of the anticoccidial feed additive nicarbazin (as dinitrocarbanilide residues) in poultry and eggs. Food Additive Contamination, 25: 32-40.

Dakpogan HB, Salifou S, Naciri M, Gbangbotche A. 2012. Comparative sensitivity of different phenotypes of free-range chicks to Eimeria tenella coccidiosis in Benin. Jour. Anim. Plant Sci., 14(3): 1978-1984.

Davies G, Genini S, Bishop S, Giuffra E. 2009. An assessment of opportunities to dissect host genetic variation in resistance to infectious diseases in livestock. Animal, 3(3): 415436.

De Gussem M. 2005. Coccidiosis control in poultry: Importance of the quality of anticoccidial premixes. Proceedings of the $9^{\text {th }}$ International Coccidiosis Conference, Foz do Iguassu.

Del Cacho E, Gallego $\mathrm{M}$, Lee $\mathrm{SH}$, Lillehoj HS, Quilez J, Lillehoj EP, Sánchez-Acedo C. 2011. Induction of protective immunity against Eimeria tenella infection using antigen-loaded dendritic cells (DC) and DC-derived exosomes. Vaccine, 29(21): 3818-3825.

Du A, Wang S. 2005. Efficacy of a DNA vaccine delivered in attenuated Salmonella typhinurium against Eimeria tenella infection in chickens. Int. J. Parasitol., 35: 777-785.

Dunnington EA, Gross WB, Martin A, Siegel PB. 1992. Response to Eimeria tenella of chickens selected for high or low antibody response and differing in haplotypes at the major histocompatibility complex. Avian Dis., 36: 49-53.

Gavora JS. 1990. Disease genetics. In Poultry Breeding and Genetics, Crawford RD (ed). Elsevier Publ. Co.: New York; 805-846

Gore TC, Long PL. 1982. The biology and pathogenicity of a recent field isolate of Eimeria praecox, Johnson, 1930. Journal of Protozoology, 29: 82-85.

Gruber A, Castanon CAB, Fernandez S, Fraga JS, Fontoura LF. 2007. COCCIMORPH: a real-time diagnostic tool based on automatic image recognition of protozoan parasites of genus Eimeria. Proceedings of the World Association for the Advancement of Veterinary Parasitology, Gent-Belgium.

Jang SI, Lillehoj HS, Lee SH, Lee KW, Park MS, Cha SR, Lillehoj EP, Subramanian BM, Sriraman R, Srinivasan VA. 2010. Eimeria maxima recombinant Gam82 gametocyte antigen vaccine protects against coccidiosis and augments humoral and cell-mediated immunity. Vaccine, 28: 2980-2985. 
Jeffers T. 1997. control of avian coccidiosis into the next millenium. In Control of Coccidiosis Into the Next Millennium, Shirley MW, Tomley FM, Freeman BM (eds). Proceedings of 7 th international coccidiosis conference, p. 16.

Johnson J, Reid WH. 1970. Anticoccidial drugs: Lesion scoring techniques in battery and floor experiments with chickens. Exp. Parasitol., 28: 30-36.

Johnson LW, Edgar SA. 1982. Responses to prolonged selection for resistance and susceptibility to acute cecal coccidiosis in the Auburn strain Single Comb White Leghorn. Poultry Sci., 61: 2344-2355.

Johnson LW, Edgar SA. 1984. Ea-A and Ea-E cellular antigen genes in Leghorn lines resistant and susceptible to acute cecal coccidiosis. Poultry Sci., 63: 1695-1704.

Kim DK, Kim CH, Lamont SJ, Keeler CL, Lillehoj JR, Lillehoj HS. 2009. Gene expression profiles of two Bcomplex disparate, genetically inbred Fayoumi chicken lines that differ in susceptibility to Eimeria maxima. Poult. Sci., 88: 1565-1579.

Lee SH, Lillehoj HS, Park DW, Jang SI, Morales A, García D, Lucio E, Larios R, Victoria G, Marrufo D, Lillehoj EP. 2009. Induction of passive immunity in broiler chickens against Eimeria acervulina by hyper immune egg yolk immunoglobulin Y. Poult Sci., 88: 562-566.

Leinsing M. 2009. Intestinal integrity: The (open) door to infection and loss of profit. European Symposium on Intestinal Integrity, Dublin.

Lillehoj HS, Ruff MD, Bacon LD, Lamont SJ, Jeffers TK. 1989. Genetic control of immunity to Eimeria tenella. Interaction of MHC genes and nonMHC linked genes influences levels of disease susceptibility in chickens. Vet
Immunol. Immunopathol., 20: 135148.

Lillehoj HS, Choi KD, Jenkins MC, Vakharia VN, Song KD, Han JY, Lillehoj EP. 2000. A recombinant Eimeria protein inducing interferon gamma production: comparison of different gene expression systems and immunization strategies for vaccination against coccidiosis. Avian Dis., 44: 379-389

Levine PP. 1939. The effect of sulfanilamide on the course of experimental avian coccidiosis. Cornell. Vet., 29: 30-320.

Levine ND. 1985. Veterinary Protozoology. Iowa State Univ Press: Ames, IA, USA; 414p.

Long PL. 1968. The effect of breed of chickens on resistance to Eimeria infections. Br. Poultry Sci., 9: 71-78.

Long PL. 1982. The Biology of Coccidia. University Park Press: Baltimore.

Martin A, Gross WB, Dunnington EA, Briles RW, Briles WE, Siegel PB. 1986. Resistance to natural and controlled exposures to Eimeria tenella: Genetic variation and 25 alloantigen systems. Poultry Sci., 65: 1847-1852.

Mathis GF. 2003. Vaccine can decrease anticoccidial resistance. World Poultry, 4: 22-23.

Mathis GF, Broussard C. 2006. Increase level of Eimeria sensitivity to Diclarzuryl after using a live coccidial vaccine. Avian Diseases, 50(3): 321324.

McDougald LR, Da Silva JML, Solis J, Braga M. 1987. A survey of sensitivity to anticoccidial drugs in 60 isolates of coccidia from broiler chickens in Brazil and Argentina. Avian Diseases, 31(2): 287 - 292.

McDougald LR. 1997. Protozoa. In: Diseases of Poultry (10th edn), Calnek BW, Barnes HJ, Beard CW, McDougald LR, 
Saif YM (eds). Iowa State University Press: Ames, IA; 865-912.

McDougald LR. 2003. Coccidiosis. Diseases of Poultry $\left(11^{\text {th }}\right.$ edn). Iowa State University Press: Ames, IA, USA.

Miller Publishing Company. 1989. Feed Additive Compendium. Miller Publishing Co.: Minneapolis, MN.

Mortier L, Huet AC, Charlier C, Daeseleire E, Delahaut P, Van Peteghem C. 2005. Incidence of residues of nine anticoccidials in eggs. Food Additive Contamination, 22: 1120-1125.

Morris GM, Gasser RB. 2006. Biotechnological advances in the diagnosis of avian coccidiosis and the analysis of genetic variation in Eimeria. Biotechnology Advances, 24: $590-603$.

Muhammad A, Shah MA, Song XS, Xu L, Yan R, Song H, Ruirui Z, Chengyu L, Li X. 2010. The DNAinduced protective immunity with chicken interferon gamma against poultry coccidiosis. Parasitol. Res., 107: 747-750.

Naciri M, De Dussem K, Fort G, Bernardet N, Nerat F, Chausse AM. 2003. Interest of anticoccidial sensitivity tests (ASTs) in the prevention of chicken coccidiosis. British Poultry Science, 44: 826-827.

Ovington KS, Alleva LM, Kerr EA. 1995. Cytokines and immunological control of Eimeria spp. International J. Parasitology, 25: 1331-1351.

Peek H, Landman W. 2006. Resistance to anticoccidial drugs of dutch avian Eimeria spp. Field isolates originating from 1996, 1999, and 2001. Avian. Pathology, 32(4): 391-401.

Pinard-van der Laan MH, Monvoisin JL, Pery P, Hamet N, Thomas M. 1998. Comparison of outbred lines of chickens for resistance to experimental infection with coccidiosis (Eimeria tenella). Poultry Sci., 77: 185-191.

Reetz J. 1993. Naturlich Mikrosporidien (Encephalitozoon cuniculi) Infecktionen bei Hühnern. Tierärztlich Praxix, 21: 429-435.

Rosenberg MM. 1941. A study of the inheritance of resistance to $E$. tenella in the domestic fowl. Poultry Sci., 20: 472.

Ruff MD, Bacon LD. 1989. Eimeria acervulina and Eimeria tenella in 15B-congenic White Leghorns. Poultry Sci., 68: 380-385.

Schmatz DM. 1997. Anticoccidial drug discovery and design. In Control of Coccidiosis Into the Next Millennium. Proceedings of $7^{\text {th }}$ international Coccidiosis Conference, Shirley MW, Tomley FM, Freeman BM, (eds). Oxford University, England; 20-21.

Sørensen JT, Edwards S, Noordhuizen J, Gunnarson S. 2006. Animal production system in the industrialized world. Scientific and Technical Review OIE, 25(2): 493- 503 .

Sujikara 1. 2000. Andrographis paniculata A paper presented at an International Conference on Tropical Agriculture for better health and environment at Kasetsart, University, Kampaengsaen, Nakornpathom, Thailand, p. 7.

Talebi A, Mulcahy G. 1995. Correlation between immune responses and oocyst production in chickens monospecifically infected with Eimeria maxima. Avian Pathol., 24: 485-495.

Tewari AK, Singh H, Sudan V, Rao JR. 2010. Recombinant surface antigen 2 (SAG 2) based serodetection of toxoplasmosis in cattle. In Proceedings of XX National Congress of Veterinary Parasitology, p 42.

Tyzzer EE, Theiler H, Jones EE. 1932. Coccidiosis in gallinaceous birds. II. A comparative study of species of Eimeria of the chicken. Am. J. Hyg., 15: 319-93. 
Vancraeynest D, Marien M, Depondt W, Nérat F, Fort G, Naciri M. 2011. Effet du decoquinate sur la coccidiose du poulet de chair déterminée par les tests de Sensibilité aux coccidiostatiques. Neuvième Journée de Recherche Avicole, Tours, 533-537.

Vermeulen A, Schaap DC, Schetters TPM. 2001. Control of coccidiosis in chicken by vaccination. Vet. Par., 100: 13-20.

Williams RB. 1995. Epidemiological studies of coccidiosis in the domestic fowl (Gallus gallus). II. Physical condition and survival of Eimeria acervulina oocysts in poultry house litter. Appl. Parasitol., 36: 90-96.

Williams RB. 1998. Epidemiological aspect of the use of live anticoccidial vaccines for chickens. International
Journal for Parasitology, 28(7): 1089 1098.

Williams RB. 1999. A compartmentalized model for the estimation of the cost of coccidiosis to the world's chicken production industry. International Journal for Parasitology, 29: 12091229.

Williams RB. 2001. Quantification of the crowding effect during infections with the seven Eimeria species of the domesticated fowl: its importance for experimental designs and the production of oocyst stocks. Int. Journ. Parsitol., 31: 1056-1069.

Youn HJ, Noh JW. 2001. Screening of the anticoccidial effect of herb extracts against Eimeria tenella. Vet. Parasitol., 96: $257-263$. 